\title{
Lengua electrónica portátil para el análisis de leche cruda basada en tecnología PSoC (Programmable System on Chip) y Android
}

\author{
Portable electronic tongue for the analysis of raw milk based on PSoC \\ (Programmable System on Chip) and Android technology
}

\author{
Álvaro Arrieta Almario ${ }^{1 *} \quad$ Oscar Fuentes Amín ${ }^{2}$
}

Recibido 14 de enero de 2015, aceptado 4 de diciembre de 2015

Received: January 14, 2015 Accepted: December 4, 2015

\begin{abstract}
RESUMEN
En el presente artículo se muestra una metodología para el desarrollo de una lengua electrónica portable, con tecnología PSoC y Android capaz de discriminar y clasificar la calidad en muestras de leche cruda. El desarrollo del dispositivo se basa en el funcionamiento del sistema gustativo humano, se conforma de una red de sensores basados, sensores electroquímicos, un sistema electrónico para el acondicionamiento de señales y una etapa de procesamiento estadístico multivariante con análisis de componentes principales y redes neuronales artificiales, acoplada a un dispositivo Android. Para el desarrollo de la etapa de acondicionamiento de señales, en la que se enfoca el presente artículo, se mostrará el desarrollo y funcionamiento de los diferentes bloques involucrados, donde el elemento central es un microprocesador PSoC 5, el cual contiene en su núcleo componentes digitales y análogos con lo que se puede crear todo el sistema de medidas electroquímicas en un simple chip y de esta forma conseguir en gran parte el ahorro de espacio al usar pocos componentes externos. Se mostrarán algunos experimentos realizados para probar el funcionamiento de cada componente del PSoC y se realizarán pruebas de comunicación vía bluetooth entre el prototipo creado y un smartphone.

Para probar el funcionamiento del sistema se mostrarán los resultados obtenidos en diferentes experimentos con el prototipo, y estos serán comparados con algunos resultados obtenidos en un equipo comercial de medidas electroquímicas, se realizarán ensayos con la aplicación Android para mostrar un resultado preliminar del funcionamiento del sistema.
\end{abstract}

Palabras clave: Lengua electrónica, leche cruda, sensores inteligentes, FREESOC, Android.

\section{ABSTRACT}

The current paper shows the development of a circuit prototype based on PSoC technology, which is used as a signal processing stage in an electronic tongue device with the ability to discriminate and classify samples of raw milk.

The electronic tongues consist of electrochemical sensors array based on conductive polymers, a signals processing stage responsible to signal data conversion, and a data processing stage based on multivariate statistical methods (principal component analysis and artificial neuronal networks). To develop the processing signals stage, it is show the development and operation of some blocks involved, where the central element is a PSoC 5 microcontroller. PSoC is an integrated circuit composed of analog and digital blocks into a configurable core, with the use of PSoC microchip, it is possible to develop a portable and small electrochemical device. Some experiments are shown to test the operation of each

1 Departamento de Biología y Química. Universidad de Sucre. Carrera 28 \# 5-267. Barrio Puerta Roja, Sincelejo, Colombia. E-mail: alvaro.arrieta@unisucre.edu.co

2 Desarrollo y Aplicación de Nuevos Materiales. Universidad Pontificia Bolivariana. Km 3 vía cerete. Montería, Colombia. E-mail: oscar.fuentesa@upb.edu.co

* Autor de correspondencia 
component of PSoC, and experiments will be made to test the bluetooth communication between the prototype and smartphone.

To test the operation of the system, the results obtained will be shown with different experiments with the prototype. Those results will be compared with those of other results obtained in a commercial electrochemical device. To show a preliminary behavior of android application, some experiments will be shown using the smartphone and the prototype.

Keywords: Electronic tongue, raw milk, smart sensor, FREESOC, Android.

\section{INTRODUCCIÓN}

Actualmente en la industria de alimentos existe una necesidad que va ligada al control de calidad de sus productos, la creciente demanda de los consumidores hace que los productores se vean obligados a implementar métodos cada vez más avanzados y precisos que garanticen esta labor de manera fiable. El desarrollo de dispositivos bioinspirados como lenguas y narices electrónicas se han convertido en una alternativa potencial en un campo donde comúnmente se aplican análisis químicos, fisicoquímicos y sensoriales, los últimos hacen la valoración por medio de los órganos de los sentidos. Hasta el momento, una de las técnicas más rigurosas para evaluar el gusto de una bebida, es mediante un panel de cata, conformado por un grupo de personas cuidadosamente preparadas y entrenadas capaces de evaluar de manera fiable, el sabor y olor de un alimento, características conocidas como organolépticas [1]. Esta valoración es un método que puede resultar costoso en dinero y tiempo, teniendo en cuenta que el proceso tiene que hacerse en condiciones ideales, los panelistas solo pueden realizar determinado número de análisis por día, se requieren óptimas condiciones de salud, los expertos resultan costosos, etc. Observando el caso particular de la leche, según el decreto 616 de 2006, expedido por el Ministerio de la protección social de Colombia, durante la recolección debe hacerse una inspección fisicoquímica y organoléptica de la leche cruda [2], lo que obliga a los productores a entrenar a los recolectores para hacer esta valoración, colocando en riesgo la salud de los mismos y a la vez aumentando la probabilidad de errores a la hora de hacer la inspección, lo que conllevaría a posibles pérdidas de lotes enteros si no se hace la valoración correcta y se mezclan muestras de leche en mal estado. Debido a esta necesidad el grupo de investigación Desarrollo y Aplicación de Nuevos Materiales (DANM) de la Universidad Pontificia
Bolivariana Montería; en conjunto con el grupo de Física del Estado Sólido de la Universidad de Valladolid ha venido desarrollando sensores basados en polímeros conductores, los quq toman señales de líquidos por medio de una técnica conocida como voltametría cíclica, que permite conseguir respuestas con gran cantidad de información del medio analizado, aumentando la capacidad de discriminación y clasificación. Aunque se han alcanzado logros en el análisis de vino [3], café [4] y cerveza [5], esta tecnología aún se encuentra en fase preliminar.

Por lo anterior, en el presente artículo se muestra el desarrollo de una etapa de adquisición y acondicionamiento de señales configurada en un chip, como parte de un prototipo de lengua electrónica portátil conformada por una red de sensores electroquímicos basados en polímeros conductores con superficies menores de $3,5 \mathrm{~mm}^{2}$, un sistema electrónico multicanal de medida y acondicionamiento de señales y un sistema de cómputo portátil, en este caso un Smartphone, equipado con un software de procesamiento estadístico que pueda ser capaz de discriminar y clasificar muestras de leche cruda.

\section{ANÁLISIS TEÓRICO}

Para el desarrollo del prototipo de lengua electrónica es importante conocer el funcionamiento del sistema gustativo humano del cual se basa esta investigación. Hasta hoy se sabe que la lengua está conformada por unas células gustativas, las que generan pulsos eléctricos al entrar en contacto con un alimento, y estos pulsos son enviados por medio de canales nerviosos al cerebro donde se da la sensación del sabor [1]. En la Figura 1 se muestra un esquema del sistema gustativo humano en paralelo con el sistema artificial desarrollado. El sistema artificial se basa en una técnica electroquímica de adquisición 


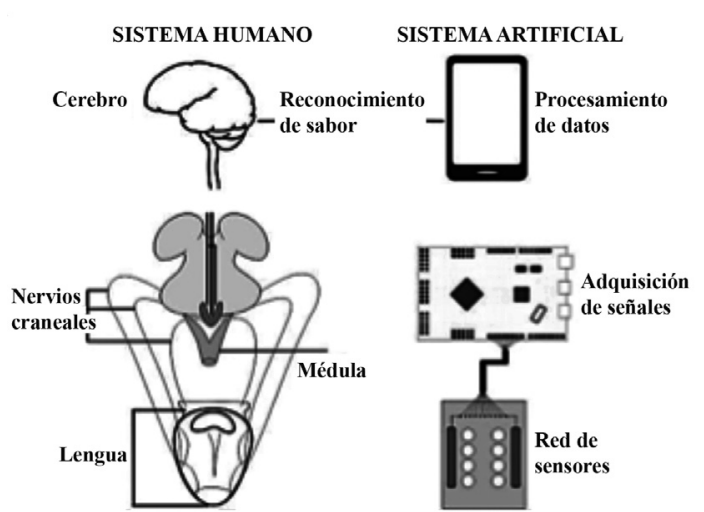

Figura 1. Esquema de sistema gustativo humano y artificial.

de señales conocida como voltametría cíclica y su comprensión es pilar para el desarrollo de lenguas electrónicas.

De la Figura 1 se puede ver que un sistema artificial de lengua electrónica lo constituyen tres etapas. La primera es una red de sensores, las que toman información de una sustancia, esta labor es cumplida biológicamente por las células gustativas de la lengua; una segunda etapa comprende la adquisición y tratamiento de las señales, lo cual equivale en el sistema biológico a la función cumplida por el tálamo, que toma los pulsos eléctricos generados en la lengua y los transmite al cerebro y, finalmente, una etapa de procesamiento de datos donde se usan métodos estadísticos multivariantes y redes neuronales artificiales, de tal forma que sea posible entrenar al sistema para que memorice los patrones, esta etapa equivale al cerebro, donde se da el reconocimiento del sabor. Para analizar la primera y segunda etapa del prototipo es importante comprender la técnica electroquímica de voltametría cíclica y el tipo de sensores utilizados en lenguas electrónicas.

\section{Sensores en lenguas electrónicas}

En una lengua electrónica los sensores constituyen la parte más importante, el objetivo principal es brindar la mayor información posible del medio analizado. Se debe tener en cuenta la influencia de algunas propiedades presentes en estos dispositivos, los cuales deben poseer características deseables como estabilidad y sensibilidad cruzada, la cual se puede entender como la capacidad de un material de responder reproduciblemente ante analitos diferentes de una solución. Generalmente esta etapa debe estar conformada por una red de sensores, ya que el medio analizado es complejo, y tener un solo sensor con selectividad cruzada no es suficiente. Sabiendo esto, la idea es desarrollar varios de estos sensores con diferentes propiedades, que ofrezcan respuestas distintas unos de otros y así conseguir una "huella dactilar" de la muestra. El material del que se constituye un sensor es determinante a la hora de encontrar estas características deseables, en la literatura se encuentran desarrollos de sensores para lenguas electrónicas a base de metales y polímeros conductores, con los cuales se han podido discriminar bebidas de acuerdo consu sabor [6-9]. Para adquirir información de una muestra se aplican técnicas como la voltametría cíclica, que se explicará con detalle más adelante; esta técnica es aplicada para conseguir señales voltamétricas mediante de tres electrodos llamados auxiliar, de referencia y de trabajo. De acuerdo al número de señales que se quieran conseguir, se conserva el mismo electrodo de referencia y auxiliar, y se determina el número de electrodos de trabajo teniendo en cuenta que deben ser de diferente naturaleza.

\section{Voltametría cíclica}

La voltametría cíclica es una técnica electroquímica muy utilizada en lenguas electrónicas; la primera basada en esta técnica fue propuesta en 1997 [6]. La técnica consiste en suministrar un potencial a una sustancia, la que varía en forma de rampa, mientras se leen las corrientes producidas durante la reacción, la variación de las corrientes con respecto a la rampa de potencial que es un gráfico conocido como voltamograma [10]. Las curvas presentes en los voltamogramas son características de las propiedades químicas de la muestra a la cual se le realice la técnica y del material del sensor que entra en contacto con ella, se puede decir que si se toman varios voltamogramas con sensores de diferentes materiales se obtiene gran cantidad de información, la que es única de la sustancia. En la Figura 2 se indica la forma en que se grafica un voltamograma.

En la Figura 3 se puede ver cómo se obtienen seis voltamogramas en una muestra de vino con sensores de diferentes materiales, de donde se observa claramente la diferencia que hay entre unas gráficas y otras dependiendo del material del cual se constituyen los electrodos de trabajo. 


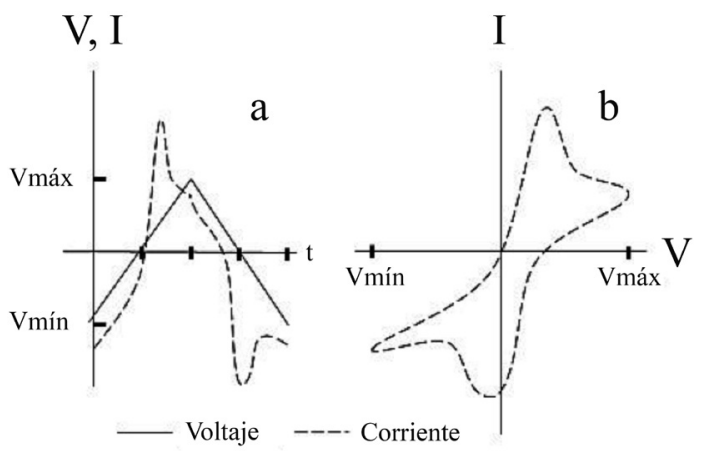

Figura 2. Gráfica de voltamograma: a) medida de corriente y voltaje; b) gráfica de corriente vs. voltaje [fuente propia].
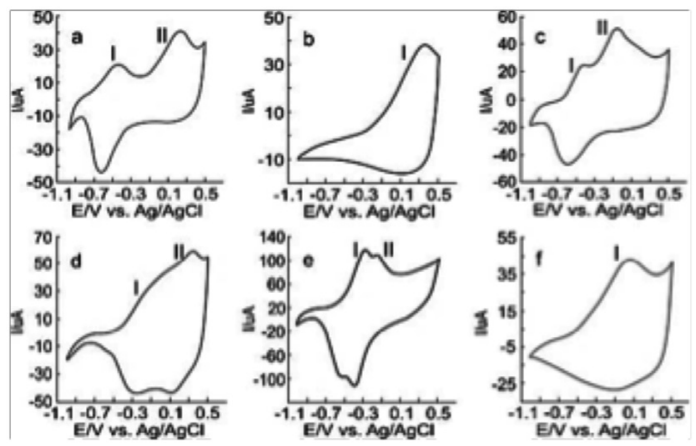

Figura 3. Señales voltamétricas de una red de sensores en vino: a) PPy/DSA, b) PPy/ TSA, c) PPy/AQDS, d) PPy/FCN, e) PPy/ PWA y f) PPy/SO4. [11].

Para la aplicación de la voltametría cíclica se utiliza un sistema de medidas electroquímicas llamado potenciostato. Este equipo es el responsable de generar la reacción en la muestra por medio de tres electrodos. El electrodo de trabajo es en forma directa donde se leen las corrientes, y de acuerdo al material del cual se encuentre constituido se obtendrán voltamogramas diferentes. El electrodo de referencia es donde se leen los voltajes, y con respecto a estas medidas se grafica la corriente para conseguir el voltamograma.

\section{Circuito de un potenciostato básico}

El potenciostato es el sistema encargado de realizar la técnica voltametría cíclica, en general el equipo puede aplicar otras técnicas de análisis electroquímico, pero en este proyecto se muestra solo el funcionamiento de la técnica que será usada como base para cumplir con el funcionamiento de la lengua electrónica. Para la aplicación de la voltametría cíclica, en primer lugar, el potenciostato genera el potencial en forma de rampa, el que será suministrado a la sustancia analizada. Además, se encarga de medir las corrientes que fluyen durante el proceso $y$, finalmente, registra los datos que luego se muestran en forma gráfica. En la Figura 4 se encuentra el circuito básico de un potenciostato.

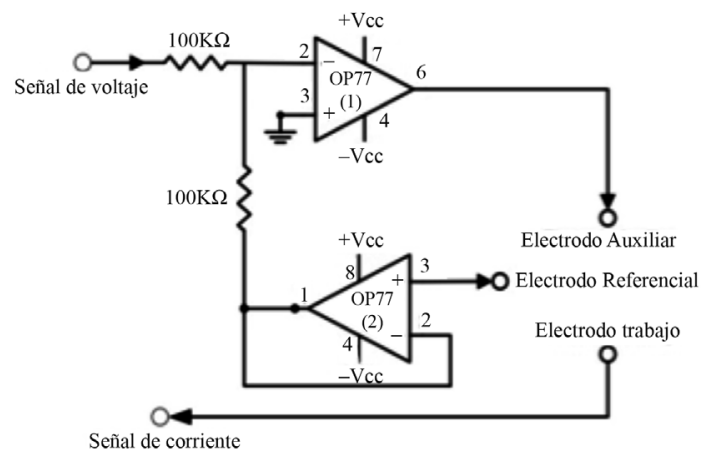

Figura 4. Circuito potenciostato básico [4].

En el circuito de la Figura 4, los tres electrodos (auxiliar, referencia y trabajo) son los sensores que entran en contacto con la sustancia analizada, en esta figura solo se muestra la parte del desarrollo que se encarga de mantener en equilibrio el potencial entre el electrodo de referencia y el de trabajo, lo que es el principal objetivo de este bloque [12]. Un diagrama completo del sistema de adquisición y tratamiento de señales se muestra en la Figura 5. Este circuito contiene un potenciostato básico, un conversor digital-análogo o DAC encargado de la generación de la rampa,

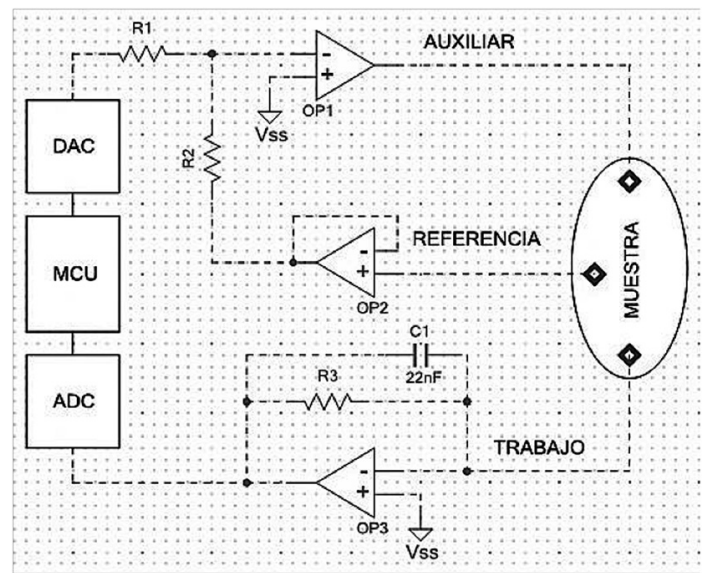

Figura 5. Bloque de adquisición y tratamiento de señales. 
un bloque conformado por el amplificador OP3, la resistencia $\mathrm{R} 3$ y el condensador $\mathrm{C} 1$, encargado de transformar las corrientes provenientes del electrodo de trabajo en voltaje, un conversor análogo-digital o ADC encargado del registro de voltajes equivalentes a las corrientes del electrodo de trabajo y un bloque de control o MCU, que comanda todo el sistema.

\section{PSoC}

Gracias a la tecnología PSoC (Programmable System on Chip) es posible el desarrollo de todo el sistema de adquisición y tratamiento de señales en un simple chip, reduciendo en gran medida el espacio del prototipo de lengua electrónica. Los PSoC's son microcontroladores desarrollados por la empresa Cypress que contienen bloques análogos y digitales dentro del mismo circuito integrado, esto los diferencia de otras familias presentes en el mercado, convirtiéndose en una buena alternativa para este tipo de desarrollos [13]. En la literatura se encuentran algunos trabajos basados en esta tecnología donde se puede observar el potencial que presentan estos componentes para el tratamiento de señales [14-16]. La familia Cypress está conformada por tres generaciones de microcontroladores, $\mathrm{PSoC} 1$, PSoC3 y PSoC5 y provee además un software gratuito llamado PSoC Creator 3.0. El desarrollo del prototipo de lengua electrónica se está implementando con la tarjeta FREESOC, el que contiene como elemento central un microprocesador PSoC5, esta tarjeta cuenta con 60 pines de propósito general E/S donde cualquiera de estos pines puede ser usado como entrada o salida análoga o digital, 8 pines especiales que pueden manejar mayor potencia, conexión USB y cuenta además con una serie de pines compatibles con Arduino.

Teniendo en cuenta las características de la familia de los PSoC's, para realizar el sistema encargado de la voltametría cíclica se usaron conversores digital-análogo o DAC, conversores análogo-digital o ADC, amplificadores operacionales, amplificadores de transimpedancia o TIA para la conversión de corriente a voltaje y componentes de comunicación serial o UART para la transmisión de los datos a la siguiente etapa.

\section{Sistema operativo Android}

La etapa encargada de procesar los datos y mostrar un resultado comprensible para el usuario se desarrolla bajo la plataforma Android. Android es un sistema operativo diseñado inicialmente para teléfonos móviles, aunque actualmente se puede encontrar en tablets, televisores, miniordenadores, etc. [17]. Este sistema operativo está basado en Linux, por lo que también se convierte en una alternativa libre, gratuita y multiplataforma para desarrollos móviles como el que se pretende. $\mathrm{La}$ inclusión de aplicaciones móviles en el desarrollo de dispositivos donde se requiere procesamiento de señales ha sido de gran utilidad, en la literatura se encuentran algunas investigaciones al respecto, se pueden mencionar trabajos como el monitoreo de glucosa en la sangre basado en espectroscopia a través de un Smartphone [18], un equipo de colorimetría para análisis químico [19], el uso de un dispositivo móvil para la determinación de $\mathrm{O}_{2}$ [20], entre otros.

Un diseño preliminar de la aplicación Android se muestra en la Figura 6, esta aplicación se conecta por bluetooth, recibe datos del sistema de acondicionamiento de señales implementado con la tarjeta Freesoc y muestra voltamogramas.

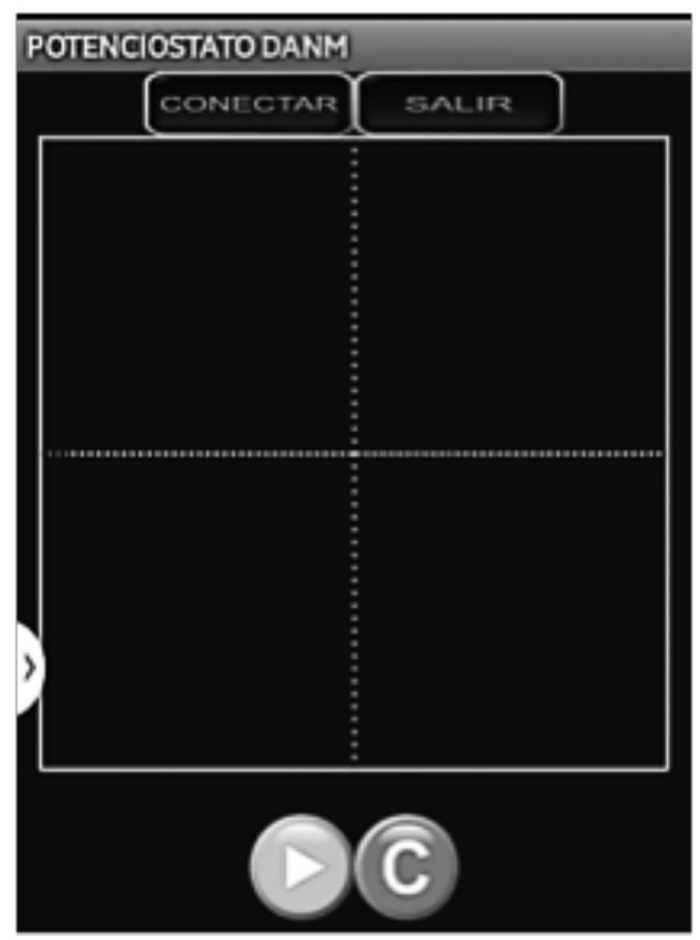

Figura 6. Aplicación Android para mostrar voltamogramas. 


\section{RESULTADOS}

Teniendo en cuenta el objetivo principal de esta investigación se han venido realizando una serie de experimentos y pruebas en cada una de las fases que lo conforman. Se sabe que la base del funcionamiento de la lengua electrónica se encuentra en la aplicación de la voltametría cíclica mediante una red de sensores poliméricos, donde se espera que cada sensor tenga una respuesta única ante la reacción producida. En la primera prueba se tomaron gráficas de corriente y voltaje en un circuito de potenciostato básico sobre un par de resistencias de $10 \mathrm{~K} \Omega$, ver Figura 7.

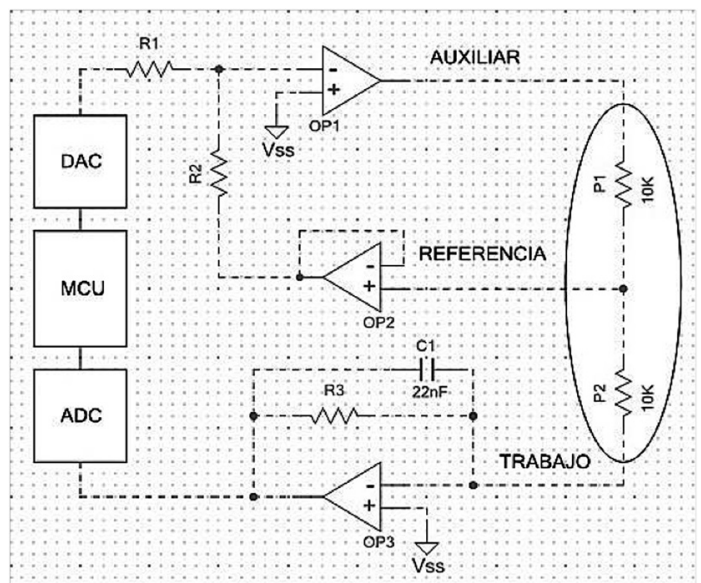

Figura 7. Prueba de voltametría cíclica en resistencias.

Para observar el voltamograma resultante se realizó una aplicación para PC en el entorno de desarrollo Processing capaz de recibir datos de un puerto COM del computador donde se conectó el prototipo y graficar las diferentes coordenadas de voltaje y corriente. La amplitud de la rampa generada fue de $-1 V$ a $1 V$ con un período de $8 s$, el proceso se hizo 40 veces para asegurar la estabilidad de la medida y con esta prueba se consiguió el voltamograma mostrado en la Figura 8.

El comportamiento observado en la resistencia en efecto es el esperado, ya que es un componente lineal, es decir, que la corriente varía en una misma proporción ante el cambio de voltaje.

La siguiente prueba consistió en la aplicación de la voltametría cíclica por medio de un potenciostato comercial PARTSTAT 2263 a una muestra de

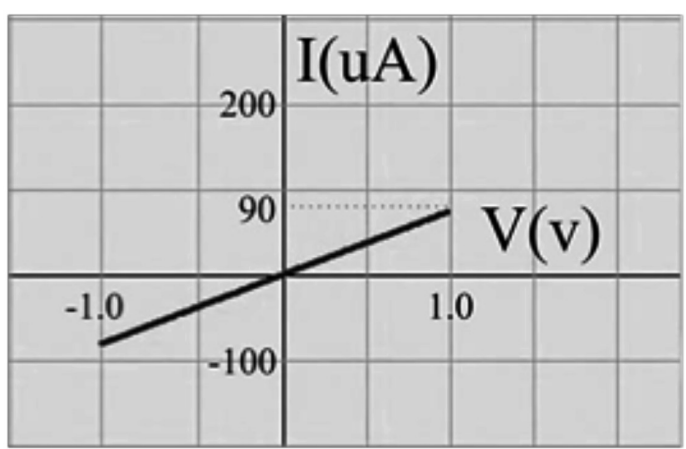

Figura 8. Voltamograma obtenido en resistencias.

ferrocianuro de potasio utilizando un electrodo de trabajo de carbono, el voltamograma resultante se puede ver en la Figura 9.

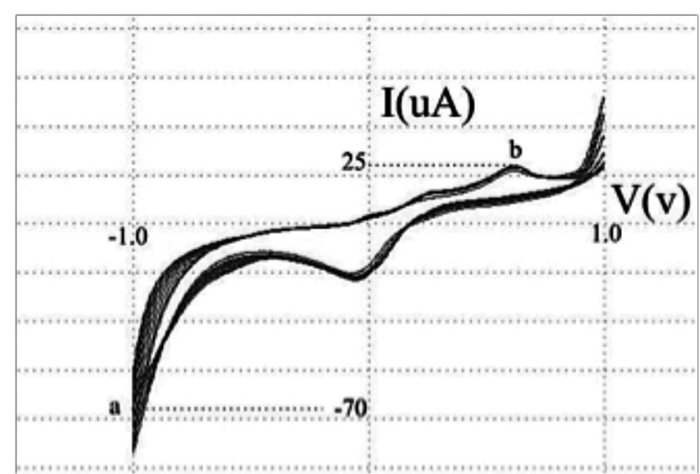

Figura 9. Voltamograma de ferrocianuro de potasio tomado en un equipo comercial.

Se realizaron 40 barridos para asegurar la estabilidad del sistema, y se tomaron medidas en dos puntos específicos durante el último ciclo, ya que durante los primeros barridos el comportamiento no fue estable.

Con el fin de verificar que el prototipo desarrollado realiza medidas válidas se hizo el experimento bajo las mismas condiciones utilizando el sistema desarrollado, de lo que se obtuvo el voltamograma mostrado en la Figura 10.

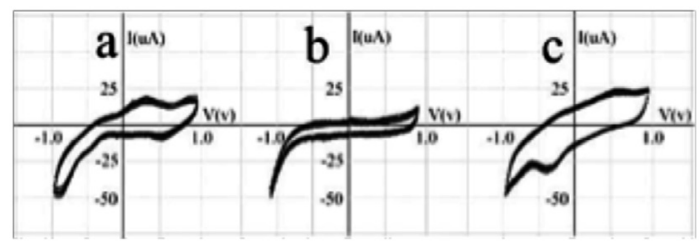

Figura 10. Voltamograma de ferrocianuro de potasio tomado en el prototipo. 
Observando las Figuras 11 y 12, a simple vista, se observa un comportamiento parecido en el resultado de ambos equipos, donde además se ve mayor estabilidad en el voltamograma de la Figura 12 ya que el sensor usado se estabilizó durante los primeros barridos de la prueba en el equipo comercial. Se puede verificar en la Tabla 1, la comparación de los puntos a y b de corriente en ambos experimentos.

Tabla 1. Comparación de medidas de corriente en ambos experimentos.

\begin{tabular}{|l|c|c|}
\hline & Punto a & Punto b \\
\hline Equipo comercial & $-70 \mu \mathrm{A}$ & $25 \mu \mathrm{A}$ \\
\hline Prototipo & $-65 \mu \mathrm{A}$ & $28 \mu \mathrm{A}$ \\
\hline Porcentaje de error & $7,14 \%$ & $10,7 \%$ \\
\hline
\end{tabular}

De la Tabla 1 se puede ver que las medidas de corriente en los puntos a y b son parecidas con un error aproximado de $7,14 \%$ y $10,7 \%$, respectivamente. Es importante resaltar que el objetivo principal de esta etapa se enfoca más que todo en la capacidad de obtener voltamogramas diferentes con un mismo material al aplicar la técnica a diversas sustancias, esto se conoce como sensibilidad cruzada. Primero se hizo una prueba sobre $\mathrm{NaCl}$, utilizando sensores con electrodos de trabajo de carbono, oro y platino respectivamente, esta prueba se repitió 50 veces para escoger uno de los tres materiales como base para el desarrollo de la red de sensores (Figura 11).

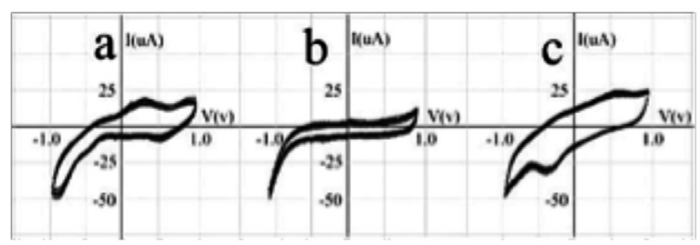

Figura 11. Voltamogramas de $\mathrm{NaCl}$ con tres materiales diferentes: a) Oro, b) carbono, c) platino.

Los voltamogramas obtenidos muestran una clara diferencia entre las medidas, esto se debe a que son materiales de diferente naturaleza. De acuerdo con lo observado durante las pruebas se tomó como base al carbono para el desarrollo de la red de sensores por su estabilidad. En el siguiente experimento se evaluó la sensibilidad cruzada, se inició el desarrollo de una aplicación para Android, la cual se instaló en un celular Samsung Galaxy Note 3, la aplicación, que se muestra en la
Figura 8, es capaz de conectarse vía bluetooth con el prototipo y graficar, de la misma manera que lo hace el programa desarrollado en Processing, los voltamogramas resultantes de la voltametría cíclica. En la Figura 12 se muestran las medidas obtenidas con un sensor de carbono en muestras de ácido láctico, lactosa y cafeína.

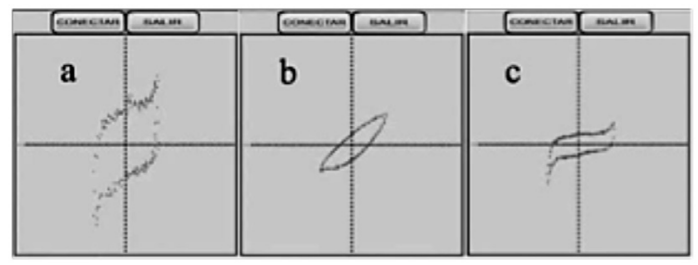

Figura 12. Voltamogramas obtenidos en la aplicación Android utilizando un sensor de carbono en: a) ácido láctico, b) cafeína, c) lactosa.

Se verificó la buena comunicación entre el prototipo y el celular Galaxy Note 3 por medio de bluetooth, donde aún no son muchos los detalles, pero se puede evidenciar la capacidad del sistema de obtener gráficas diferentes y únicas para cada sustancia analizada, esto indica que el carbono es un material con buena sensibilidad cruzada, y es precisamente el comportamiento ideal para ser tomado como base en la etapa de procesamiento estadístico donde el sistema tiene que discriminar y clasificar muestras de leche cruda.

\section{CONCLUSIONES}

Con el desarrollo de este prototipo se pudo comprobar que la tecnología PSoC ofrece las características necesarias para el desarrollo de un equipo portátil que aplique la voltametría cíclica, siendo una buena alternativa para el procesamiento de pequeñas señales y la aplicación de lengua electrónica en el sector lechero.

Por otro lado, con la red de sensores conformada por oro, platino y carbono, se obtuvieron respuestas diferentes en cada material frente a $\mathrm{NaCl}$, lo que es esencial para una lengua electrónica, ya que a la hora de adquirir la mayor información posible de una sustancia se requiere el uso de una red de sensores con estas características.

Finalmente se pudo comprobar la capacidad del sistema para responder con un patrón único frente a 
sustancias de diferente naturaleza, esta información obtenida por los sensores es importante a la hora de discriminar las sustancias según su sabor.

\section{AGRADECIMIENTOS}

Los autores agradecen el soporte económico brindado por el Departamento Administrativo de Ciencia, Tecnología e Innovación - Colciencias y la Universidad Pontificia Bolivariana-Montería. Contrato RC No 0401-2013.

\section{REFERENCIAS}

[1] B. Lindemann. "Taste reception". Physiological Reviews. Vol. $76 \mathrm{~N}^{\circ} 3$, pp. 719766. Julio 1996. ISSN: 1522-1210. DOI: 719-766.

[2] Ministerio de la protección social. "Decreto número 616”. Bogotá D.C. 2010.

[3] V. Parra, M. Rodríguez, Á. Arrieta, J. Fernández and J. De Saja. "Electronic tongue based on chemically modified electrodes and voltammetry for the detection of adulterations in wines". Sensors and Actuators. Vol. 118, pp. 448-453. May, 2006. ISSN 0925-4005. DOI: 10.1016/j.snb.2006.04.043.

[4] Á. Arrieta Almario y R. Tarazona Cáceres. "Sistema multipotenciostato basado en instrumentación virtual". Ingeniería Investigación y Tecnología. Vol. $15 \mathrm{~N}^{\mathrm{o}} 3$, pp. 321-337. Julio 2014. ISSN: 1405-7743.

[5] Á. Arrieta, C. Blanco, M. Rodríguez, J. De Saja and D. Nimubona. "Prediction of bitterness and alcoholic strength in beer using an electronic tongue". Food Chemistry. Vol. 123, pp. 642-646. Diciembre 2010. ISSN: 0308-8146. DOI: 10.1016/j. foodchem.2010.05.006.

[6] F. Winquist, P. Wide and I. Lundström. "An electronic tongue based on voltammetry". Analytica Chimica Acta. Vol. 357, pp. 21-31. Diciembre 1997. ISSN: 0003-2670 DOI: 10.1016/S0003-2670(97)00498-4.

[7] P. Ivarsson, S. Holmin, N. Höjer, C. KrantzRülcker and F. Winquist. "Discrimination of tea by means of a voltammetric electronic tongue and different applied waveforms". Sensors and Actuators B: Chemical. Vol. 76, pp. 449-454. Junio 2001. ISSN: 0925-4005. DOI: 10.1016/S0925-4005(01)00583-4.
[8] K. Woertz, C. Tissen, P. Kleinebudde and J. Breitkreutz. "Taste sensing systems (electronic tongues) for pharmaceutical applications". International Journal of Pharmaceutics. Vol. 417, pp. 256-271. Septiembre 2011. ISSN: 0378-5173. DOI: 10.1016/j.ijpharm.2010.11.028.

[9] Á. Arrieta, M. Rodríguez y J. De Saja. "Aplicación de una lengua electrónica voltamétrica para la clasificación de vinos y estudio de correlación con la caracterización química y sensorial". Química Nova. Vol. 33 $\mathrm{N}^{\circ}$ 4, pp. 787-793. 2010. ISSN: 0100-4042.

[10] J. Gónzalez. "Técnicas y métodos de laboratorio clínico". Masson, S.A. 2da Edición. Barcelona, España, pp. 199. 2005. ISBN: 84-458-1321-8.

[11] Á. Arrieta, O. Fuentes y A. Jaramillo. "Sistema portátil de sensores inteligentes (lengua electrónica) para el análisis de alimentos". Revista Investigaciones Aplicadas. Vol. $8 \mathrm{~N}^{\circ} 1$, pp. 30-36. Junio 2014. ISSN: 2011-0413.

[12] M. Orazem and B. Tribollet. "Electrochemical Impedance Spectroscopy". Wiley. New Jersey, United States, pp. 101. 2008. ISBN: 978-0-470-04140-6.

[13] M. Mar, B. Sullam and E. Blom. "An architecture for a configurable mixedsignal device". Solid-State Circuits, IEEE Journal of. Vol. $38 \mathrm{~N}^{\circ} 3$, pp. 565-568. Marzo 2003. ISSN: 0018-9200. DOI: 10.1109/ JSSC.2002.808298.

[14] J. Cabrera y J. Velasco. "Implementación de Filtros Análogos Usando PSoCs/FPAAs". Revista Colombiana de Física. Vol. $40 \mathrm{~N}^{\circ} 2$, pp. 399-401. Julio 2008. ISSN: 0120-2650.

[15] A. Jackson, C.T. Moritz, J. Mavoori and T.H. Lucas. "The neurochip BCI: towards a neural prosthesis for upper limb function". Neural Systems and Rehabilitation Engineering, IEEE Transactions on. Vol. $14 \mathrm{~N}^{\circ} 2$, pp. 187190. Junio 2006. ISSN: 1534-4320. DOI: 10.1109/TNSRE. 2006.875547.

[16] J. Cabrera y J. Velasco. "Diseño e Implementación de un Sistema Embebido para el Procesamiento de Señales Ultrasónicas". Revista Colombiana de Física. Vol. $43 \mathrm{~N}^{\circ} 1$, pp. 100-104. Abril 2011. ISSN: 0120-2650.

[17] M. Báez, Á. Borrego, J. Cordero, L. Cruz, M. Gonzáles, F. Hernández, D. Palomero, J. Rodriguez, D. Sanz, M. Saucedo, P. Torralbo 
y Á. Zapata. "Introducción a Android". E.M.E. Madrid, España, pp. 121. 2012. ISBN: 978-84-96285-39-5.

[18] V. Dantu, J. Vempati and S. Srivilliputhur, "Non-invasive blood glucose monitor based on spectroscopy using a smartphone". Engineering in Medicine and Biology Society (EMBC), 2014 36th Annual International Conference of the IEEE. Chicago, Estados Unidos. 2014.

[19] N. Lopez, V. Curto, M. Erenas, F. BenitoLópez, D. Diamond, A. Palma and L. Capitan
"Smartphone-based simultaneous $\mathrm{pH}$ and nitrite colorimetric determination for paper microfluidic devices". Analytical chemistry. Vol. $86 \mathrm{~N}^{\circ} 19$, pp. 9554-9562. Agosto 2014. ISSN: 2156-8278. DOI: 10.1021/ac5019205

[20] N. López-Ruiz, A. Martínez-Olmos, I.M. Pérez de Vargas-Sansalvador, M.D. FernándezRamos, M.A. Carvajal, L.F. Capitan-Vallvey and A.J. Palma. "Determination of $\mathrm{O}_{2}$ using colour sensing from image processing with mobile devices". Sensors and Actuators B: Chemical. Vol. 171-172, pp. 938-945. 2012. 